\title{
Application of 3D Scanning in Prosthetic \& Orthotic Clinical Practice
}

\author{
Jiri ROSICKY ${ }^{1,2,3}$, Ales GRYGAR ${ }^{1,2}$, Petr CHAPCAK ${ }^{1,2}$, Tomas BOUMA ${ }^{1,2}$, Jan ROSICKY,2 \\ ${ }^{1}$ Invent Medical Group, Ostrava, Czech Republic; \\ ${ }^{2}$ ING corporation, Frydlant nad Ostravici, Czech Republic; \\ ${ }^{3}$ Ortopedicka protetika Frydek-Mistek, Frydek-Mistek, Czech Republic \\ DOI: $10.15221 / 16.088$ http://dx.doi.org/10.15221/16.088
}

\begin{abstract}
This presentation is focused on application of 3D body scanning that represents a standard part of digital processes that we use in our prosthetic \& orthotic clinical practice. The description of digital workflows such as CAD/CAM process (3D scanning, CAD/CAM, CNC milling) or Direct Digital Manufacturing process (3D scanning, computational modelling, 3D printing) will be done. We will give an overview of the application of different scanners for P\&O clinical practice.
\end{abstract}

Keywords: 3D scanning, prosthetics, orthotics, anaplastology, computational modelling, CAD/CAM, 3D printing, Direct Digital Manufacturing

\section{Introduction}

$3 \mathrm{D}$ scanning is a part of digital workflows in a modern prosthetic and orthotic clinical practice. We use either CAD/CAM process (3D scanning, CAD/CAM, CNC milling) or Direct Digital Manufacturing process (3D scanning, computational modelling, 3D printing) [1], [2], [3], [4].

\section{Digital workflows in Prosthetics \& Orthotics}

\subsection{CAD/CAM workflow}

CAD/CAM workflow is represented by $3 \mathrm{D}$ scanning, computational design (CAD), preparation of digital manufacturing data (CAM) and $\mathrm{CNC}$ milling.
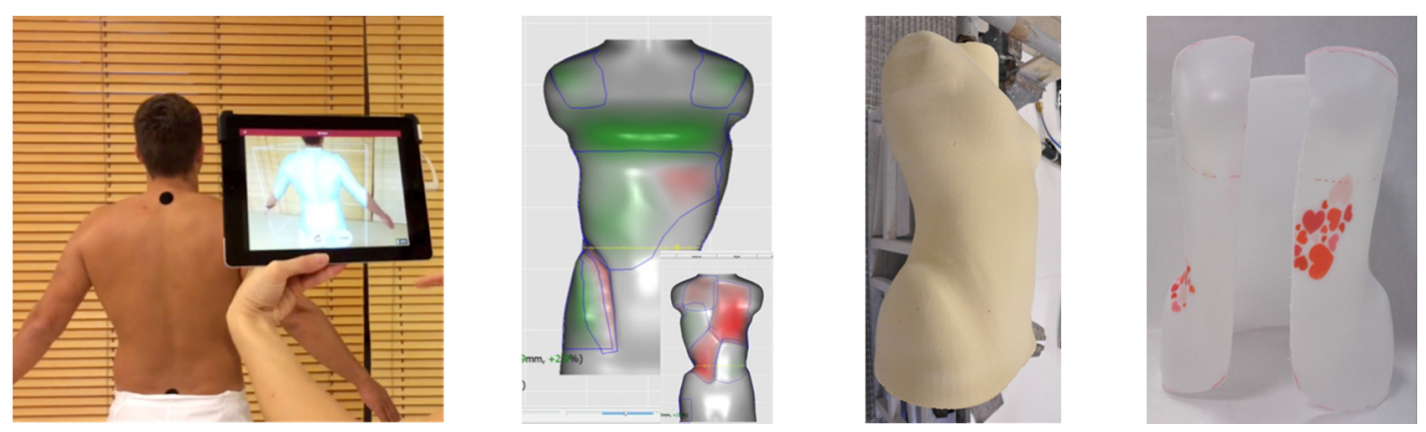

Fig. 1. CAD/CAM workflow - 3D scanning, CAD desing, CNC milled model, orthotic product

The output of CAD/CAM process is a CNC milled model from a hard PUR foam. This model is used for manual manufacturing of a custom-made orthosis or a prosthetic socket by laminating or thermoplastic forming. CAD/CAM process is a combination of digital technologies and manual operations.

\subsection{Direct Digital Manufacturing process}

Direct Digital Manufacturing (DDM) represents a fully digital workflow of 3D scanning, computational modelling and 3D printing. 

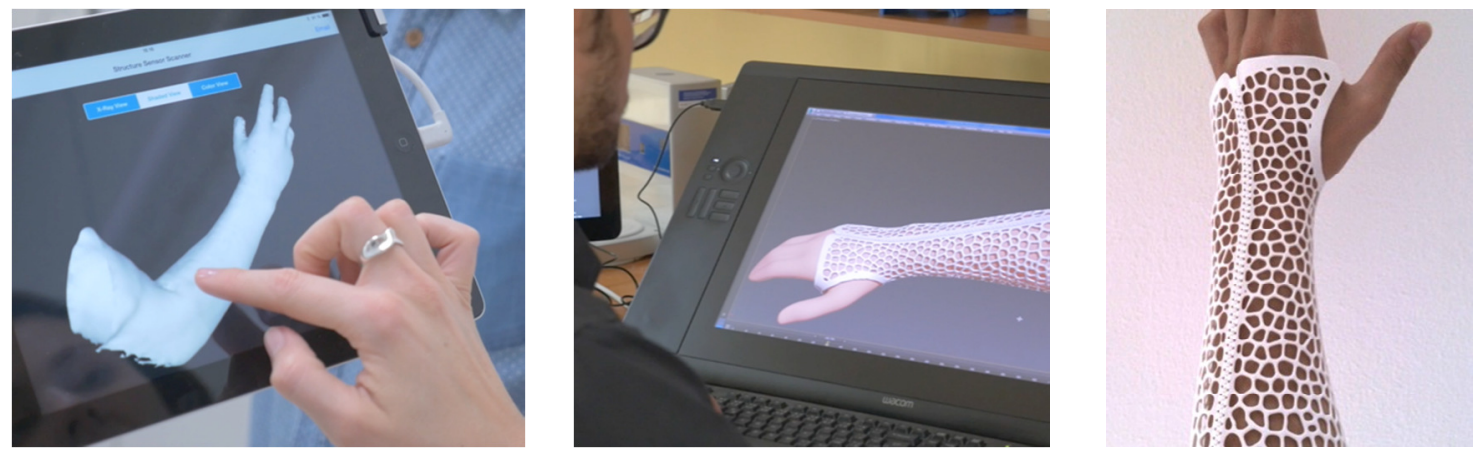

Fig. 2. DDM workflow - 3D scanning, computational modelling, 3d printed orthosis

The output of DDM process is a 3D printed product (orthosis, prosthesis or their parts) or 3D printed mold for manufacturing the prostheses (e.g. silicone cosmetic hands).

\section{General requirements on 3D scanners in Prosthetics \& Orthotics}

There are different 3D scanners that can be used in P\&O clinical practice. Their application is dependent on many parameters: accuracy and resolution, scanning area, portability, time of preparation/scanning/post-processing, scanning principle, ease of operation, price etc. Scanners for head and face, trunk, limb and foot are used in our clinical practice daily.

There is not a single scanner that would fulfil all the requirements for a clinical application. The combination of individual requirements in each $\mathrm{P} \& \mathrm{O}$ branch determines the choice of 3D scanner for a clinical application. There is an overview of general requirements on 3D scanners for main prosthetic and orthotic applications in Table 1.

Table 1. Overview of general requirements on $3 D$ scanners in $P \& O$

\begin{tabular}{|c|c|c|c|c|c|c|c|c|}
\hline & 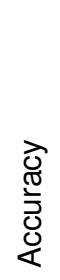 & 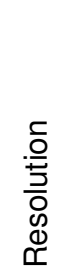 & 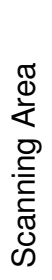 & 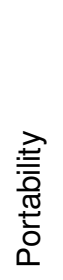 & 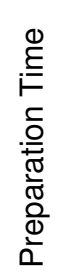 & 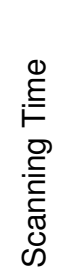 & 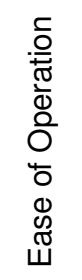 & 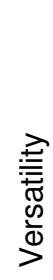 \\
\hline Insoles & $x$ & $x$ & $x$ & $x$ & $x$ & $x$ & $x x x$ & $x$ \\
\hline Orthoses & $x$ & $x$ & $\mathbf{x x}$ & $x x$ & $x$ & $\mathbf{x x}$ & $x x x$ & $\mathbf{x x}$ \\
\hline Prostheses & $x$ & $x$ & $\mathbf{x x}$ & $\mathbf{x x}$ & $x$ & $\mathbf{x x}$ & $x x x$ & $x x$ \\
\hline Epitheses & $\mathbf{x x}$ & $x x x$ & $x$ & $x$ & $x$ & $x x$ & $x x x$ & $x$ \\
\hline
\end{tabular}

\section{Overview of 3D scanners in Prosthetics \& Orthotics}

We are using different types of 3D scanners in our clinical practice. Here is a very brief overview of 3D scanners divided into groups: low-end mobile scanners $(<1.000 \$)$, high-end mobile scanners $(<25.000 \$)$ and high-end special scanners (>25.000\$). 


\subsection{Low-end mobile scanners $(<1.000 \$)$}

We are using the following mobile 3D scanners in this range: Sense (Cubify) and Structure (Occipital).

Table 2. Sense

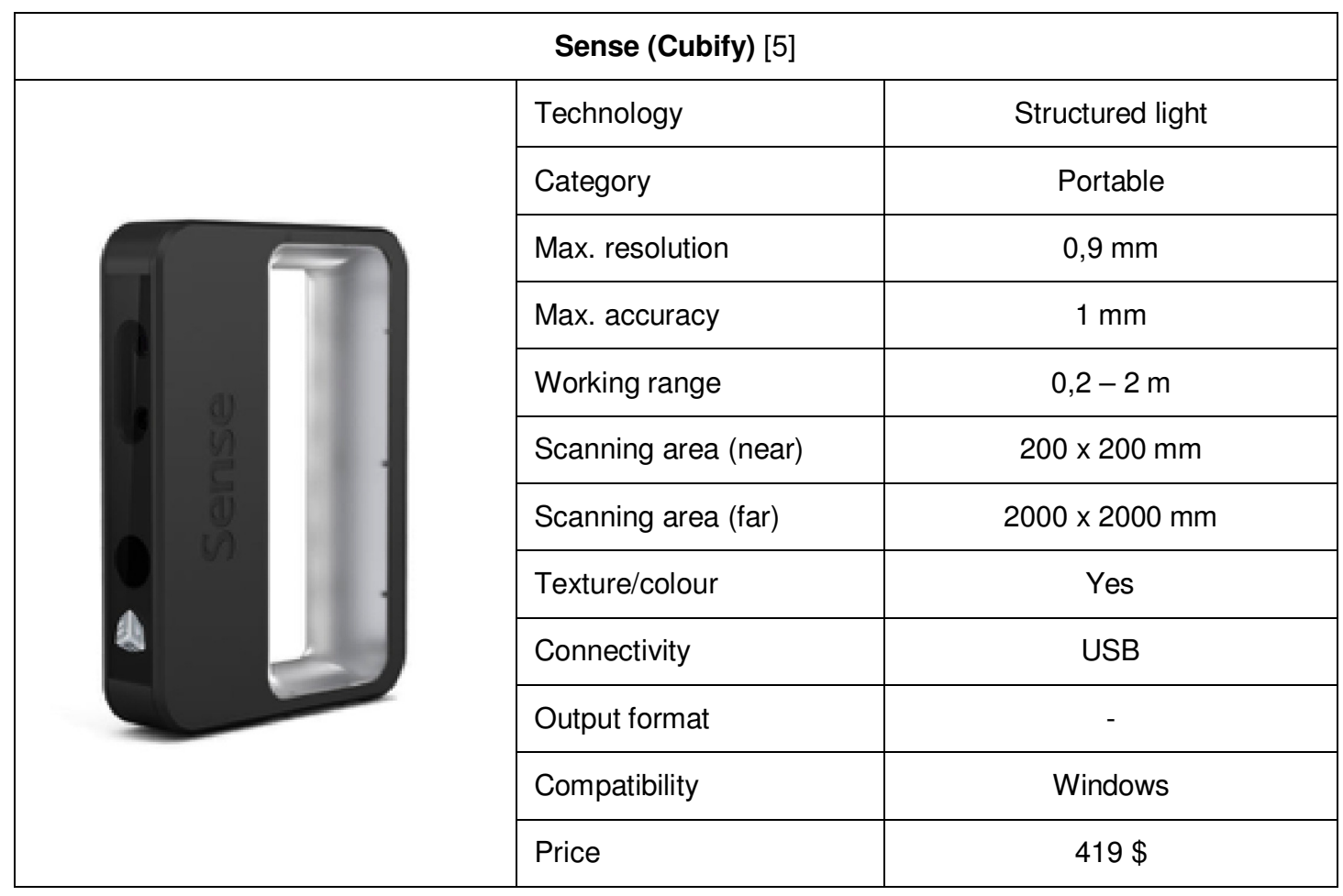

Table 3. Structure Sensor

\begin{tabular}{|l|l|c|}
\hline \multicolumn{3}{|c|}{ Structure Sensor (Occipital) [6] } \\
\hline & Technology & Structured light \\
\cline { 2 - 3 } & Category & Portable \\
\cline { 2 - 3 } & Max. resolution & - \\
\hline & Max. accuracy & 0,5 mm \\
\hline & Working range & 0,4 - 3,5 m \\
\hline & Scanning area (near) & - \\
\hline & Scanning area (far) & Yes \\
\hline & Texture/colour & USB, WiFi \\
\hline & Connectivity & OBJ, PLY, STL, VRML, WRL \\
\hline & Output format & iOS \\
\hline & Compatibility & 379 \$ \\
\hline
\end{tabular}

Low-end mobile scanners are used for less precise applications in $\mathrm{P} \& \mathrm{O}$, e.g. for spinal/limb braces, prosthetic covers. These scanners are easy to use and they are affordable. 


\subsection{High-end mobile scanners $(<25.000 €)$}

We are using the following mobile 3D scanners in this range: Artec EVA (Artec), Go!SCAN 3D (Creaform) and zSnapper Portable (VIALUX).

Table 4. Artec EVA

\begin{tabular}{|c|c|c|}
\hline \multicolumn{3}{|c|}{ Artec EVA (Artec) [7] } \\
\hline \multirow{12}{*}{ 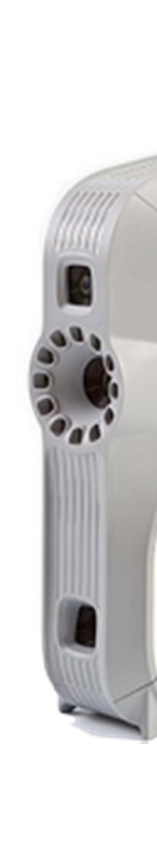 } & Technology & Structured light \\
\hline & Category & Portable \\
\hline & Max. resolution & $0,5 \mathrm{~mm}$ \\
\hline & Max. accuracy & $0,1 \mathrm{~mm}$ \\
\hline & Working range & $0,4 \mathrm{~m}$ \\
\hline & Scanning area (near) & $214 \times 148 \mathrm{~mm}$ \\
\hline & Scanning area (far) & $536 \times 371 \mathrm{~mm}$ \\
\hline & Texture/colour & Yes \\
\hline & Connectivity & USB \\
\hline & Output format & $\begin{array}{l}\text { AOP, ASCII, E57, OBJ, PLY, } \\
\text { PTX, STL, WRL, XYZRGB }\end{array}$ \\
\hline & Compatibility & Windows \\
\hline & Price & $19.800 \$$ \\
\hline
\end{tabular}

Table 5. GoISCAN 3D

\begin{tabular}{|c|c|c|}
\hline \multicolumn{3}{|c|}{ Go!SCAN 3D (Creaform) [8] } \\
\hline \multirow{12}{*}{ c } & Technology & Structured light \\
\hline & Category & Portable \\
\hline & Max. resolution & $0,5 \mathrm{~mm}$ \\
\hline & Max. accuracy & $0,1 \mathrm{~mm}$ \\
\hline & Working range & $0,4-0,65 \mathrm{~m}$ \\
\hline & Scanning area (near) & $380 \times 380 \mathrm{~mm}$ \\
\hline & Scanning area (far) & - \\
\hline & Texture/colour & No \\
\hline & Connectivity & USB \\
\hline & Output format & $\begin{array}{c}\text { DAE, FBX, MA, OBJ, PLY, STL, } \\
\text { TXT, WRL, X3D, X3DZ, ZPR }\end{array}$ \\
\hline & Compatibility & Windows \\
\hline & Price & Approx. $20.000 \$$ \\
\hline
\end{tabular}


Table 6. zSnapper Portable

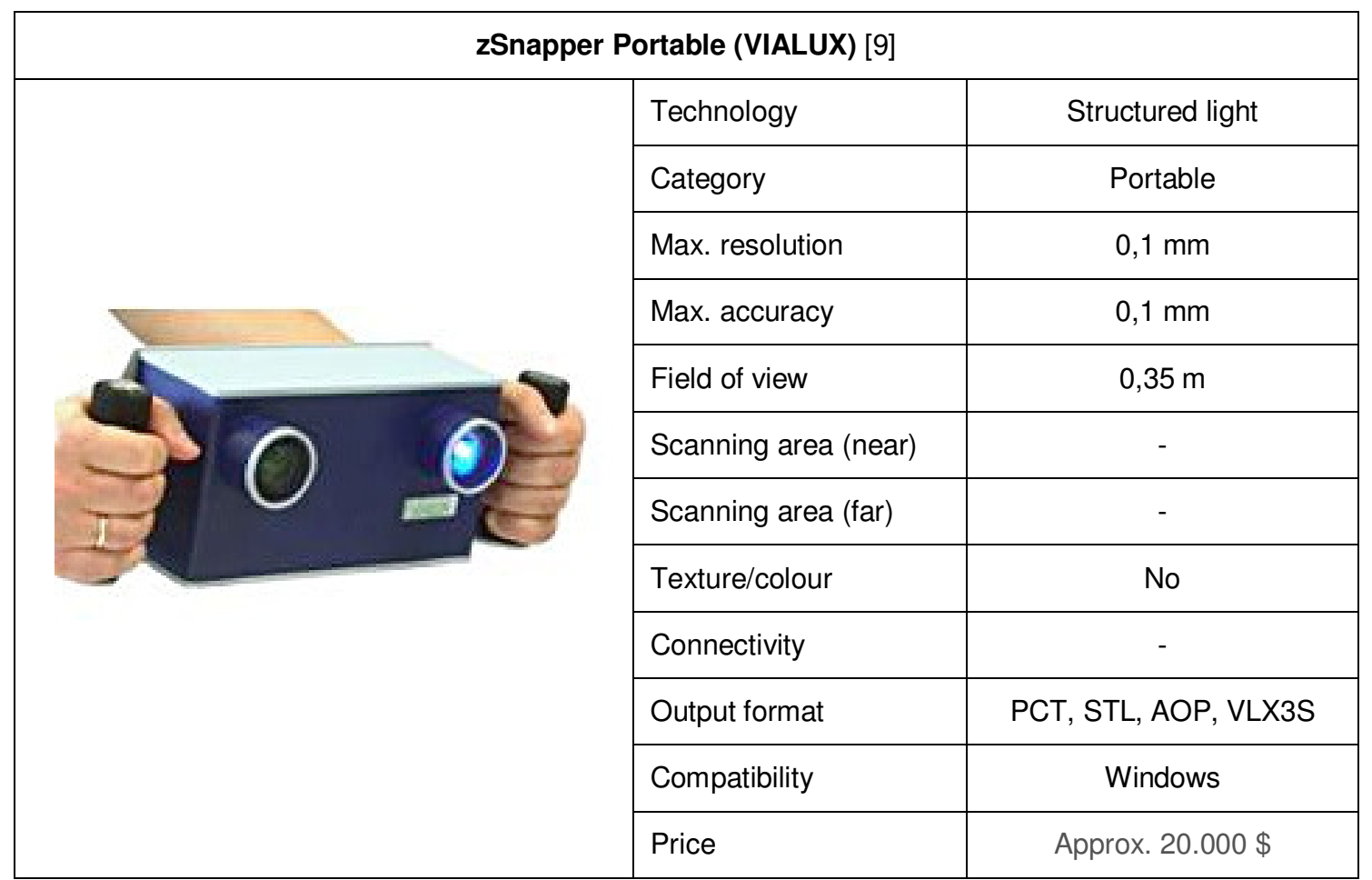

High-end mobile scanners can be used for the same applications in P\&O as low-end mobile scanners (spinal/limb braces, prosthetic covers) but with a better quality output. The main benefit of these scanners is especially for more precise applications, e.g. head and face scanning, scanning for prosthetic sockets and epitheses.

We have found high-end mobile scanners very versatile for P\&O applications and still easy to use. The cost of these scanners represents a limit for usage in small P\&O workshops.

\subsection{High-end special scanners $(>25.000 €)$}

We are using the following mobile 3D scanners in this range: DI3D FCS-100 (Dimensional Imaging) and ATOS Core 135 (GOM). These scanners belong to industrial scanners and they have special applications in P\&O.

DI3D FCS-100 scanner (Dimensional Imaging) is used for face scanning. The photogrammetry technology enables a very fast scanning (4 digital cameras are synchronized) and the result is a 3D scan of the face with a color texture. It is possible to take scans with eyes open of the scanned person. These characteristics are very important for facial scanning and clinical application. An overview of technical data is described in Table 7.

ATOS Core 135 scanner (GOM) is used for scanning of very precise casts of body parts. These precise casts are manufactured from plaster based on silicone or alginate impressions. This scanner is used for high-definition silicone prostheses (facial and limb silicone prostheses). An overview of technical data is described in Table 8.

We have found high-end special scanners very useful for special P\&O applications - anaplastology (silicone prostheses and epitheses) and facial masks (burn mask, protection mask). The cost of these scanners is a very limiting factor even for big P\&O workshops. 
Table 7. DI3D FCS-100 (Dimensional Imaging)

\begin{tabular}{|c|c|c|}
\hline \multicolumn{3}{|c|}{ DI3D FCS-100 (Dimensional Imaging) [10] } \\
\hline & Technology & Photogrammetry \\
\hline & Category & Industrial \\
\hline & Max. resolution & - \\
\hline & Max. accuracy & - \\
\hline & Working range & $0,8-0,9 m$ \\
\hline & Scanning area (near) & $250 \times 350 \mathrm{~mm}$ \\
\hline & Scanning area (far) & - \\
\hline & Texture/colour & Yes \\
\hline & Connectivity & - \\
\hline & Output format & STL, OBJ \\
\hline & Compatibility & Windows \\
\hline & Price & Approx. $30.000 \$$ \\
\hline
\end{tabular}

Table 8. ATOS Core 135 (GOM)

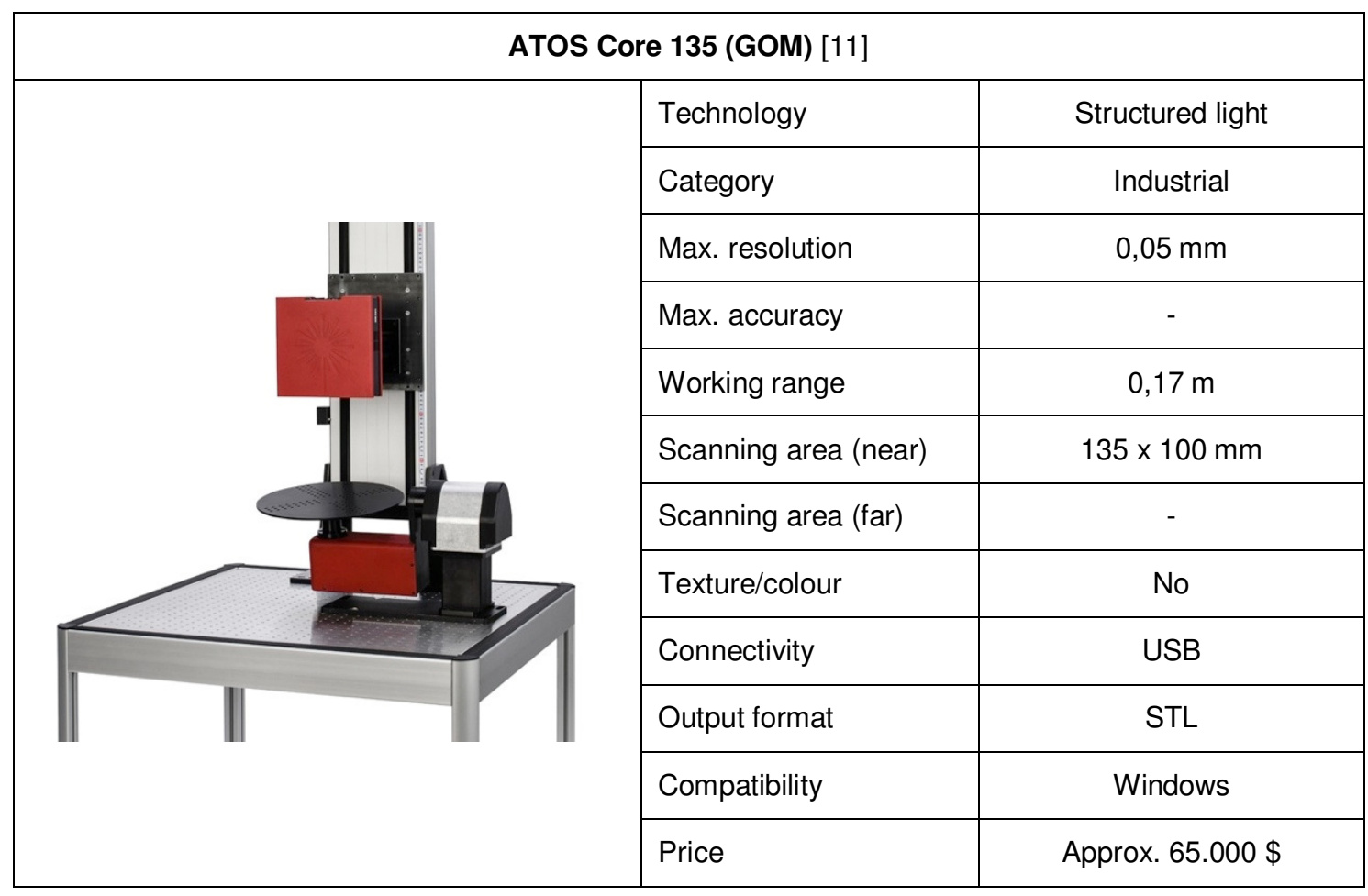

A comparison of all 3D scanners that we are using in our $P \& O$ clinical practice is done in Table 9. 
Table 9. Comparison of $3 D$ scanners for $P \& O$ applications

\begin{tabular}{|c|c|c|c|c|c|c|c|}
\hline & 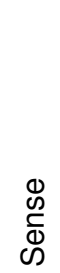 & 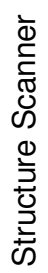 & 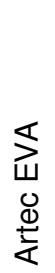 & 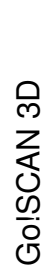 & $\begin{array}{l}\frac{0}{0} \\
\frac{0}{0} \\
\frac{0}{0} \\
\frac{2}{\Phi} \\
\frac{0}{0} \\
\frac{0}{0} \\
\stackrel{N}{N}\end{array}$ & 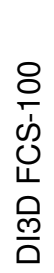 & 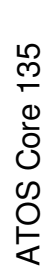 \\
\hline Insoles & $x$ & $x$ & $x$ & $x$ & $x$ & & \\
\hline Limb \& Trunk Orthoses & $x$ & $x$ & $x$ & $x$ & $x$ & & \\
\hline Head \& Face Orthoses & & & $x$ & $x$ & $x$ & $x$ & \\
\hline Prostheses - Cosmetic covers & $x$ & $x$ & $x$ & $x$ & $x$ & & \\
\hline Prostheses - Sockets & & & $x$ & $x$ & $x$ & & \\
\hline Epitheses & & & $x$ & $x$ & $x$ & $x$ & $x$ \\
\hline \multicolumn{8}{|c|}{ x - useful application } \\
\hline
\end{tabular}

\section{Clinical application of 3D scanners in Prosthetics \& Orthotics}

\subsection{Head \& Face}

3D scanners are used for the following P\&O applications:

- Cranial helmets (remoulding, protective)

- Burn compression masks

- Sport protective masks

- Facial silicone prostheses

The examples of head \& face 3D scans are in the following pictures (Fig. 3. - Fig. 6.).

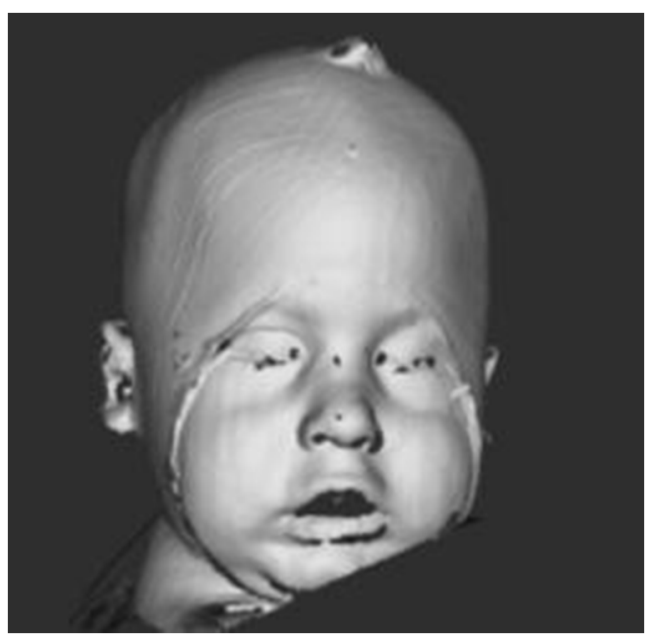

Fig. 3. 3D scan for a helmet (zSnapper portable)

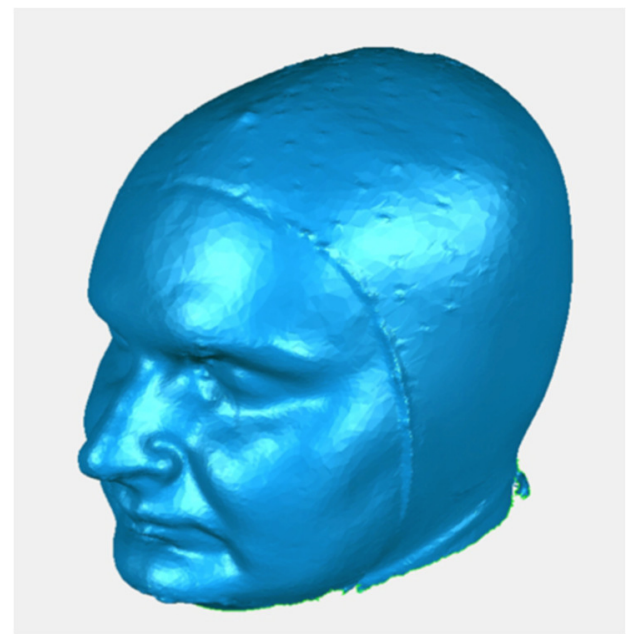

Fig. 4. 3D scan for a face mask (Artec EVA) 


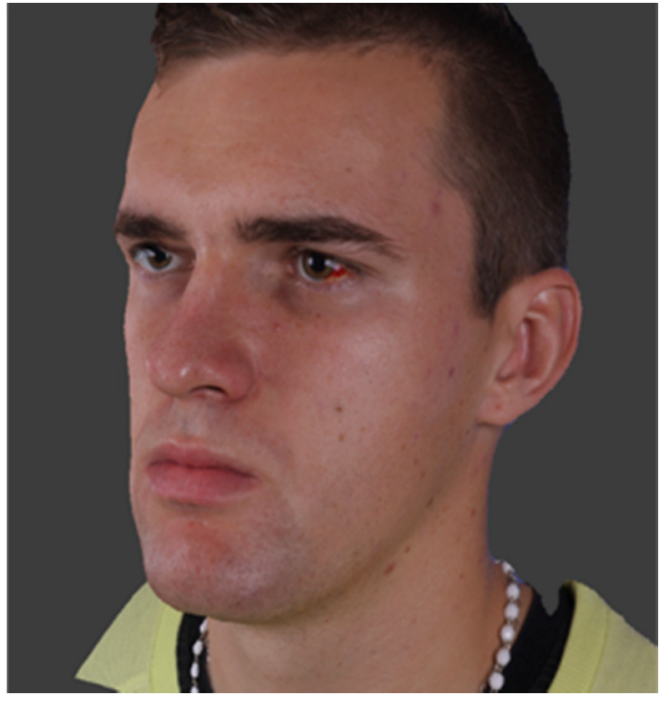

Fig. 5. 3D scan for a face mask (DI3D FCS-100)

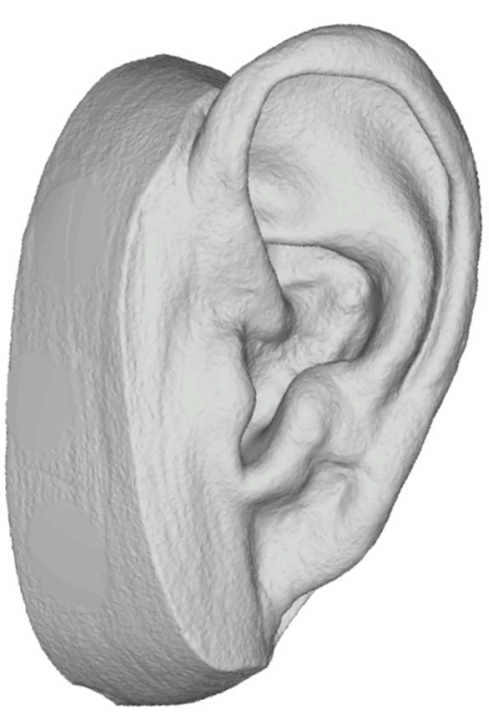

Fig. 6. 3D scan for a silicone prosthesis (ATOS Core)

\subsection{Limb \& Trunk}

3D scanners are used for the following P\&O applications:

- Spinal braces

- Upper limb \& lower limb orthoses

- Upper limb \& lower limb prostheses

The examples of limb \& trunk 3D scans are in the following pictures (Fig. 7. - Fig. 8.).

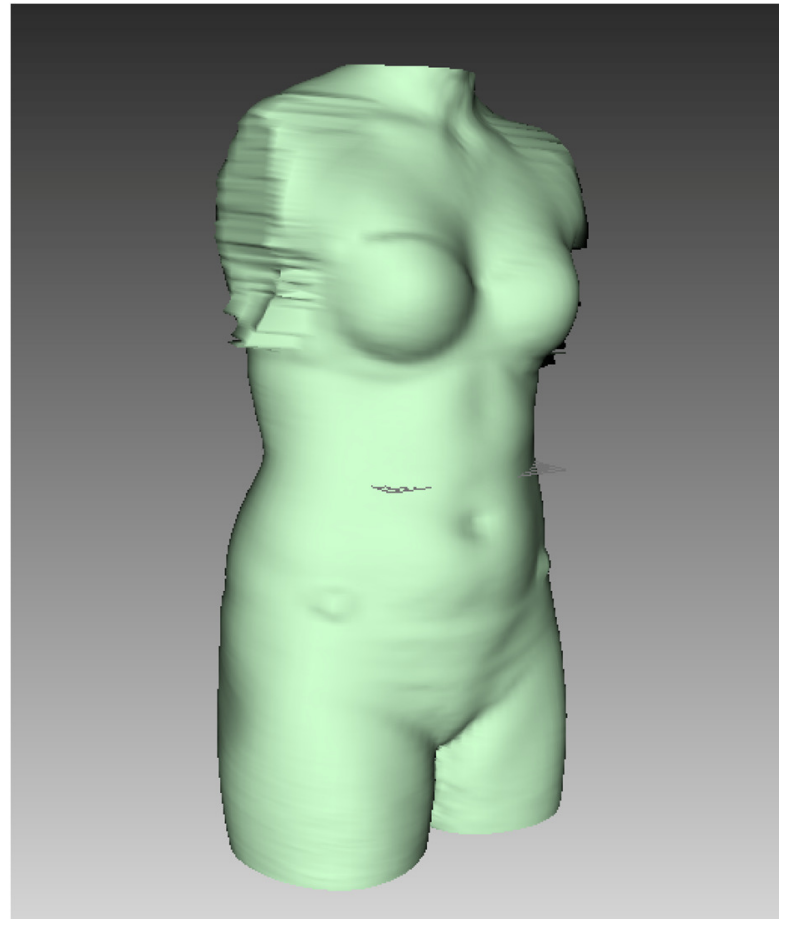

Fig. 7. 3D scan for spinal brace (Structure Sensor)

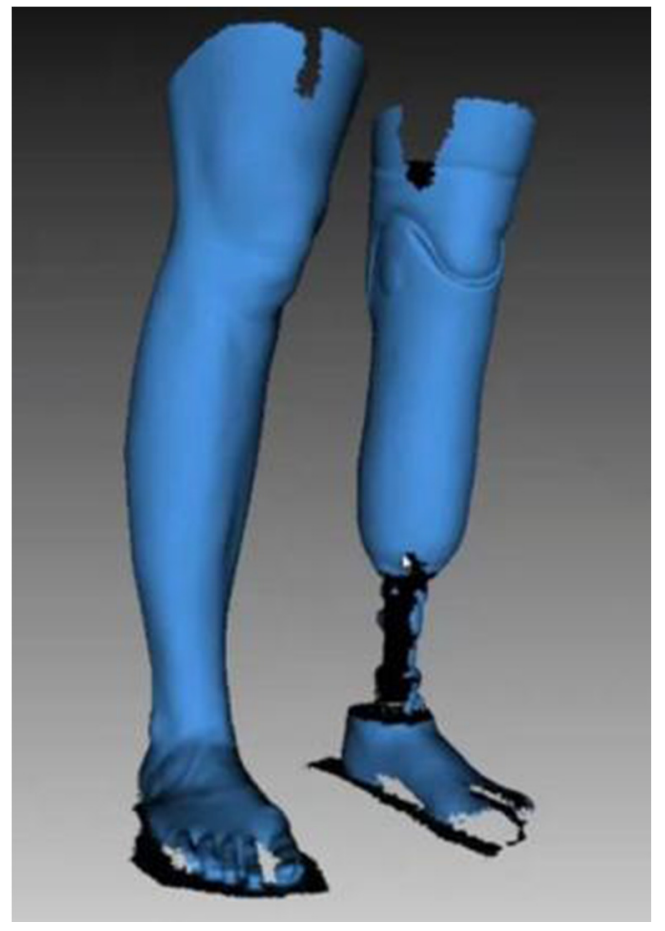

Fig. 8. 3D scan for a prosthetic cover (Artec EVA) 


\subsection{Foot}

3D scanners are used for the following P\&O applications:

- Foot orthoses (insoles)

- Silicone prostheses for partial foot amputations

The examples of foot 3D scans are in the following pictures (Fig. 9. - Fig. 10.).

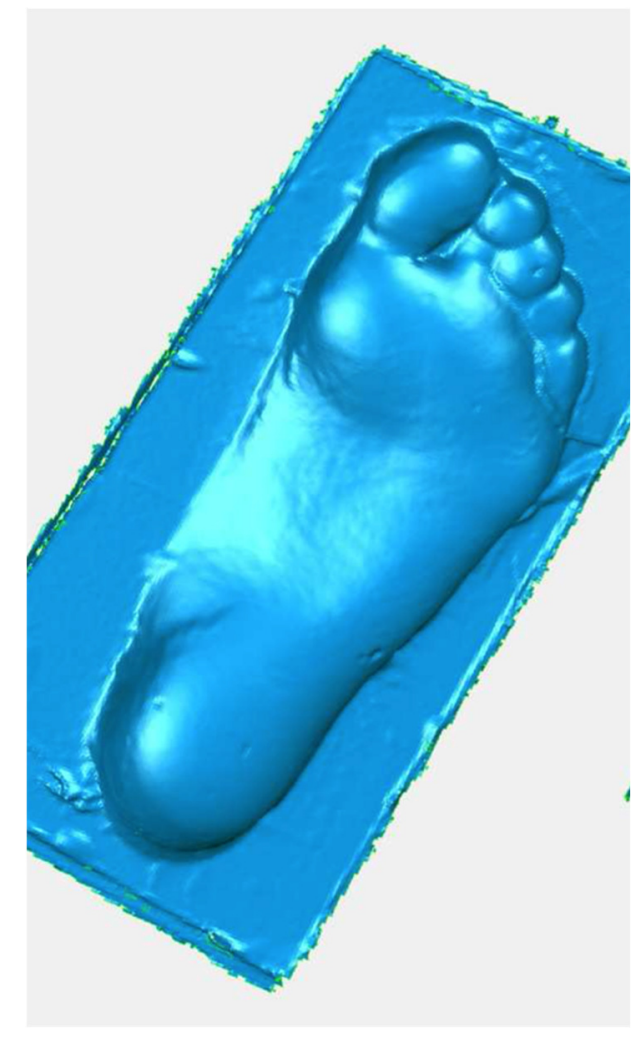

Fig. 9. 3D scan for an insole (ARTEC EVA)

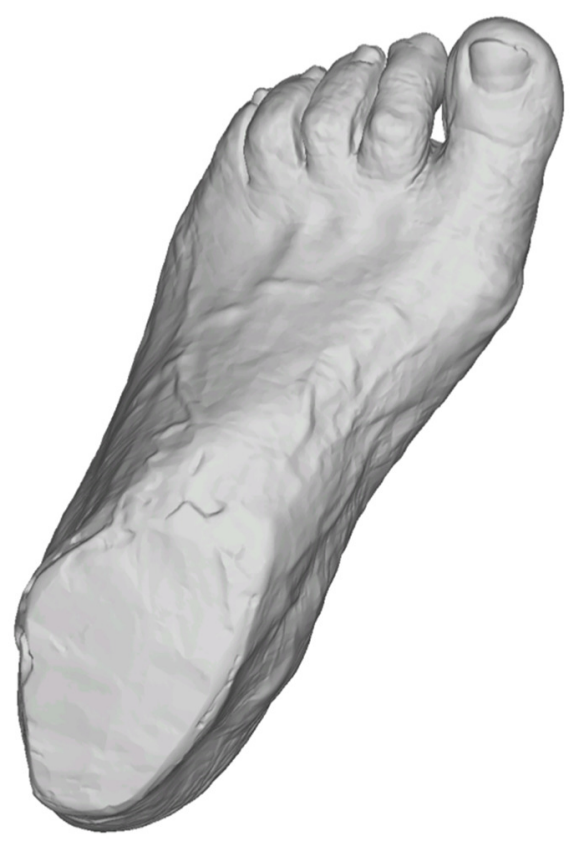

Fig. 10. $3 D$ scan for a silicone foot prosthesis (ATOS Core)

\section{Results}

3D scanning is a part of digital workflows in a modern prosthetic and orthotic clinical practice. 3D scanners for head \& face, trunk, limb and foot are used in our clinical practice daily. There are different 3D scanners that can be used in $\mathrm{P} \& \mathrm{O}$ clinic. There is not one scanner that would fulfil all the requirements for all clinical application in $\mathrm{P} \& \mathrm{O}$.

Low-end mobile 3D scanners $(<1.000 \$)$ are used for less precise applications in P\&O, e.g. for spinal/limb braces, prosthetic covers. These 3D scanners are easy to use and they are cost affordable.

High-end mobile 3D scanners ( $<25.000 \$)$ could be used for the same applications in P\&O as low-end mobile scanners (spinal/limb braces, prosthetic covers) but with better quality output. The main benefit of these scanners is especially for more precise applications, e.g. head \& face scanning, scanning for prosthetic sockets and epitheses. We have found high-end mobile 3D scanners very versatile for $\mathrm{P} \& \mathrm{O}$ applications and still easy to use. The cost of these scanners represents a limit for usage in small P\&O workshops.

High-end special 3D scanners $(>25.000 \$)$ are very useful for special P\&O applications anaplastology (silicone prostheses \& epitheses) and facial masks (burn masks, protection masks). The cost of these 3D scanners is a very limiting factor even in big P\&O workshops. 


\section{References}

[1] J. Boleslavska et. al. "Facial Epitheses", in Journal of Otorinolaryng. a Foniat., No. 3, 2014, pp. 193-197, Praha.

[2] D. Palousek et. al. "Pilot study of the wrist orthosis design process" in Rapid Prototyping Journal, No. 20/1, 2014, pp. 27-32, Emerald Group Publishing Limited [ISSN 1355-2546]

[3] D. Palousek et. al. "Use of digital technologies for nasal prosthesis manufacturing" in Prosthet Orthot Int. Journal, 2013, 0309364613489333

[4] D. Koutny et. al.: "3D Digitalization of the Human Body for Use in Orthotics and Prosthetics" in World Academy of Science, Engineering and Technology, International Journal of Medical Science and Engineering, Vol.72, No.12, 2012.

[5] 3D scanner Sense, http://www.3dsystems.com, accessed 2016.

[6] 3D scanner Structure Sensor, http://structure.io, accessed 2016.

[7] 3D scanner Artec EVA, http://www.artec3d.com, accessed 2016.

[8] 3D scanner Go!SCAN, https://www.goscan3d.com, accessed 2016.

[9] 3D scanner zSnapper portable, http://www.vialux.de, accessed 2016.

[10] 3D scanner DI3D FCS-100, http://www.di4d.com, accessed 2016.

[11] 3D scanner ATOS Core 135, http://www.gom.com, accessed 2016. 